\title{
Arrhythmia Classification via Time and Frequency Domain Analyses of Ventricular and Atrial Contractions
}

\author{
Irena I Jekova $^{1 *}$, Todor V Stoyanov ${ }^{1}$, Ivan A Dotsinsky ${ }^{1}$ \\ ${ }^{1}$ Institute of Biophysics and Biomedical Engineering, Bulgarian Academy of Sciences, Sofia, \\ Bulgaria
}

\begin{abstract}
Atrial fibrillation $(\mathrm{AF})$ is associated with significant risk of heart failure and consequent death. Its episodic appearance, the wide variety of arrhythmias exhibiting irregular AF-like RR intervals and noises accompanying the ECG acquisition, impede the reliable AF detection. Therefore, the Computing in Cardiology Challenge 2017 organizers encourage the development of methods for classification of short, single-lead ECG as AF, normal sinus rhythm (NSR), other rhythm (OR), or noisy signal. The arrhythmia classification module presented in this paper involves procedures for QRS detection and classification, $P$-waves detection, feature calculation in the time and frequency domains. The applied decision rule is a classification tree. The scores over the training (test subset) [whole test] datasets are: $F_{N S R}=0.82(0.81)$; $F_{A F}=0.62(0.61) ; F_{O R}=0.61(0.53), F 1=0.68(0.65)[0.64]$.
\end{abstract}

\section{Introduction}

Atrial fibrillation (AF) is the most common sustained cardiac tachyarrhythmia, with incidence increasing from $0.5 \%$ at age of $40-50$ years up to $5-15 \%$ for 80 years old people [1]. It is characterized by uncoordinated atrial activation and deterioration of atrial mechanical function, associated with significant risk of heart failure and consequent death [2].

There are three general approaches for AF detection:

- Atrial activity analysis associated with investigation of the TQ interval for presence of multiple P-waves [3,4] or absence of P-waves [5];

- Ventricular response analysis associated with RR intervals investigation via assessment of their median absolute deviation [6], irregularity [7], sample entropy [8], etc.;

- Combination of independent data from the atrial and ventricular contractions analyses $[9,10]$.

A comparative study of AF detection methods [11] highlights the techniques based on analysis of RR interval irregularity as the most robust against noise, providing the highest sensitivity and specificity, while the combination of RR and atrial activity analysis assures the highest positive predictive value.

The ECG analyses for AF detection are performed either in the time [3-10] or in the frequency domain, where the dominant AF frequency is usually assessed over a signal with extracted QRS-T complexes [12,13].

Considering the episodic appearance of AF, the wide variety of arrhythmias exhibiting irregular AF-like RR intervals and the diverse noises accompanying the ECG acquisition, the organizers of the Computing in Cardiology Challenge 2017 encouraged the promotion of methods for classification of short single lead ECG as $\mathrm{AF}$, normal sinus rhythm (NSR), other rhythm (OR), or noisy signal (NOISE). This paper presents a module for discrimination between AF, NSR, OR and NOISE based on time and frequency domain analyses of ventricular and atrial contractions.

\section{Challenge database}

The Challenge database contains single-lead ECGs recorded via AliveCor device at $300 \mathrm{~Hz}$ sampling rate. The recordings are separated to two independent datasets:

- Training dataset, which contains 8528 ECGs annotated in four classes according to the rhythm type (5050 'Normal', 738 'AF', 2456 'Other rhythm') and signal quality (284 noisy ECGs). The duration of the ECG strips is in the range (9-61s), approximately the same for all class annotation.

- Test dataset, containing 3658 ECGs unavailable to the public for the purpose of scoring.

\section{Method}

The module for discrimination between AF, NSR, OR and NOISE in a single-lead ECG is developed in Matlab (MathWorks Inc.). It implements a preprocessing stage, including high-pass filter with cut-off frequency $1 \mathrm{~Hz}, 2$ comb filters with first zeros $50,60 \mathrm{~Hz}$; feature extraction procedures in the time and frequency domain; noise detection rule; and rhythm analysis block (Figure 1). 


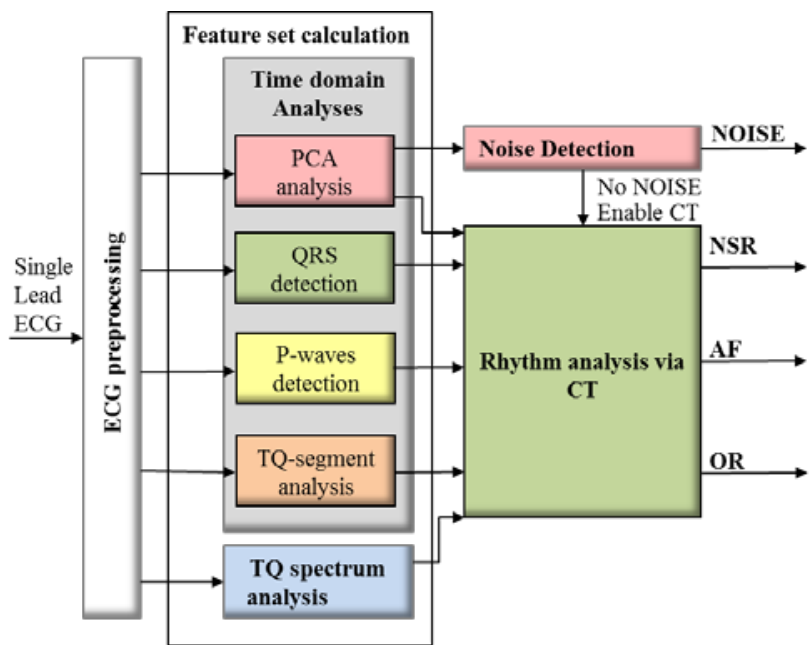

Figure 1. Flow chart of the module for discrimination between AF, NSR, OR and NOISE.

\subsection{Feature extraction procedures}

\section{QRS detection}

The time domain analysis starts with QRS detection based on location of steep edges and sharp peaks, followed by heartbeat classification via RR-interval and waveform analysis. Detailed description of the procedure could be found in [14]. The stability of the detected ventricular contractions is assessed via calculation of:

- Mean, minimal, maximal values and standard deviation of the QRS amplitudes and RR-intervals for all ventricular beats (VB), as well as only for the normal sinus beats $(\mathrm{N})$ : MeanAmp_VB, MinAmp_VB, MaxAmp_VB, StdAmp_VB, MeanAmp_N, MinAmp_N, $M a x A m p \_N$, StdAmp_N, MeanRR_VB, MinRR_VB, $\operatorname{MaxRR\_ VB,} \quad S t d R R \_V B, \quad M e a n R R \_N, \quad \operatorname{Min} R R \_N$, $M a x R R \_N$, StdRR_N. These features are considered as appropriate for discrimination between NSR, AF, OR;

- $\mathrm{N}$ beats ratio, expected to present distinct values for NSR and OR: NBeats(\%) $=100 *$ Number $_{\mathrm{N}} /$ Number $_{\mathrm{VB}}$;

- Probability the rhythm to be AF based on assessment of the RR irregularity:

$$
\mathrm{AF}(\%)= \begin{cases}80 & \text { if }(\text { MaxRR_VB }- \text { MeanRR_VB })>200 \mathrm{~ms} \\ 60 & \text { if }(\text { MaxRR_VB }- \text { MeanRR_VB })>160 \mathrm{~ms} \\ 0 & \text { if }(\text { MaxRR_VB }- \text { MeanRR_VB })<80 \mathrm{~ms} \\ 40 & \text { otherwise }\end{cases}
$$

\section{$P$-waves detection}

The P-waves are detected via in-house developed procedure, applying simple time and amplitude criteria over each RR interval.

The stability of the detected atrial contractions is assessed via calculation of:

- Mean, minimal, maximal values and standard deviation of the P-waves amplitudes and PP intervals: MeanAmpP, MinAmpP, MaxAmpP, StdAmpP, MeanPPint, MinPPint, MaxPPint, StdPPint;
- Mean value and standard deviation of the P-waves number in each RR interval: MeanPcountRRint, StdPcountRRint.

\section{PCA analysis}

Principal component analysis (PCA) is used for assessment of the ECG signal beat-to-beat irregularity. The PCA is applied over the PQRST and the TR segments wrapped respectively between:

- PQRST: (QRSindex-0.25*SR) and

$$
\text { (QRSindex }+0.5 * S R * \sqrt{\text { MeanRR_VB/SR) }}
$$

- TR: (QRSindex - 0.8*MeanRR_VB) and (QRSindex $100 \mathrm{~ms}$ ), enclosing the $\mathrm{T}$-wave, $\mathrm{P}$-wave and atrial fibrillation waves (if present).

Two features representing the mean value of the standard deviation between the samples of the respective segments and the corresponding samples of its first PCA vector (Figure 2) are calculated:

- MeanStdPQRST after normalization of all PQRST segments towards the maximal absolute value of PQRST first PCA vector. This feature is expected to be appropriate for NSR vs. OR discrimination, as well as for noise detection;

- MeanStdTR, supposed to be representative for noisy signals.

\section{TQ-segment analysis}

Considering that the AF influences the waveforms appearing between the T-wave end and the Q-wave by mimicking ventricular fibrillation (VF) like patterns, two analysis procedures typical for VF detection are applied over the ECG signals after elimination of the QRS-T segments (i.e. over the TQ intervals) and the following features are calculated in the time domain:

- TQ-signal complexity (C) [15], expected to present high values for AF signals. $\mathrm{C}$ is calculated for the first, the middle and last 6s segment of the TQ signal and the median value is further considered;

- TQ-signal leakage after application of a steep rejection filter over 3s segments of the TQ signal adjusted at its main frequency [16]. The following features are considered MeanLeakTQ, MinLeakTQ, MaxLeakTQ, StdLeakTQ, supposed to be low for AF signals;

The frequency domain analysis of the TQ segments is performed over $4 \mathrm{~s}$ non-overlapping intervals and it includes calculation of:

- Dominant frequency (DF) [13], corresponding to the maximum power in the range (3-15) Hz: MeanDF, MinDF, MaxDF, StdDF;

- Regularity index [13] (RI = spectrum area within $\mathrm{DF} \pm 0.75 \mathrm{~Hz} /$ spectrum area within $3-15 \mathrm{~Hz}$ ), quantify the sharpness of the dominant peak: MeanRI, MinRI, MaxRI, StdRI;

- Spectral width at level $0.8^{*}$ Spectral amplitude(DF): MeanSpecWidth_08, Min SpecWidth_08, Max SpecWidth_08, Std SpecWidth_08. 

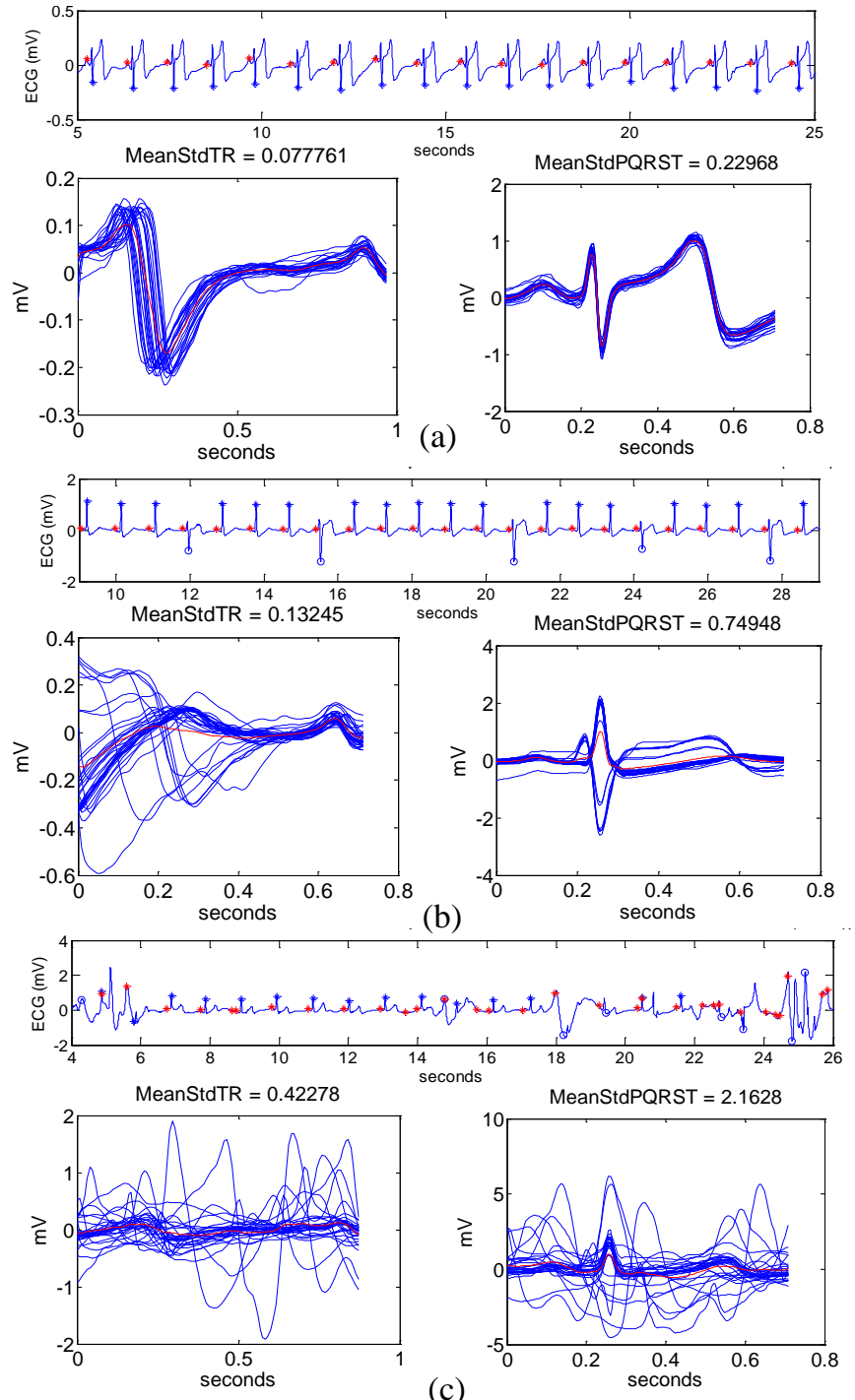

Figure 2. Examples of NSR (a), OR (b), NOISE (c), the respective TR, PQRST segments (in blue) and TR, PQRST first PCA vectors (in red). The detected normal beats are marked with blue asterisks $\left({ }^{*}\right)$, ectopic beats with blue circles (o), and P-waves with red asterisks $(*)$.

\subsection{Decision making}

The noise detection is performed via a single threshold rule MeanStdTR $\geq 0.5$, which minimally influences the correct detection of the NSR, AF, OR rhythms (see the distribution in Figure 3). The three rhythm types are discriminated via a classification tree (CT) model, generated and pruned by means of the statistical package Statistica (v. 12.3, Dell Inc.), using the following settings:

- Three categories of the classification variable according to the rhythm type: NSR, AF, OR;

- Splitting based on Gini index;

- Optimal prior probabilities for NSR vs. AF vs. OR 0.4 vs. 0.2 vs. 0.4 ;

- Pruning criterion based on misclassification rate.
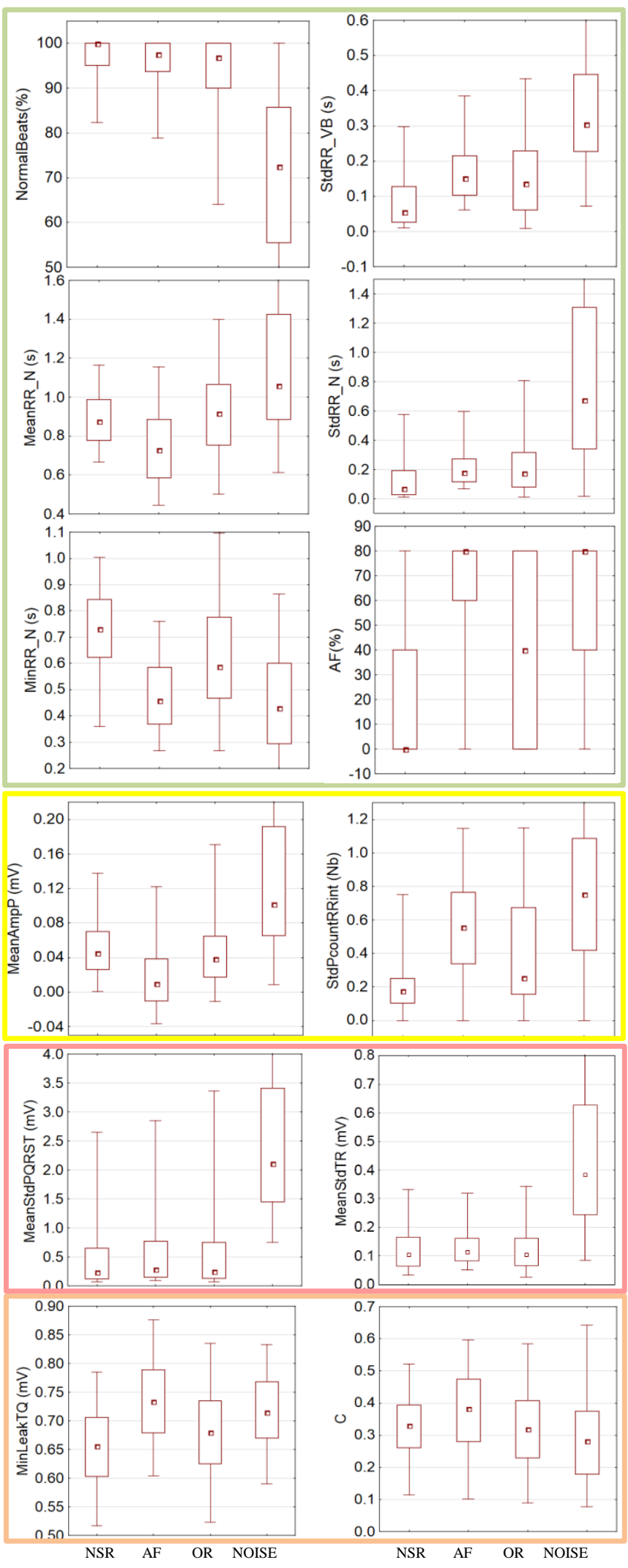

Figure 3. Distribution of time-domain features included in CT: QRS detector (wrapped in green), P-wave detector (yellow), PCA analysis (red), QT analysis (orange) 

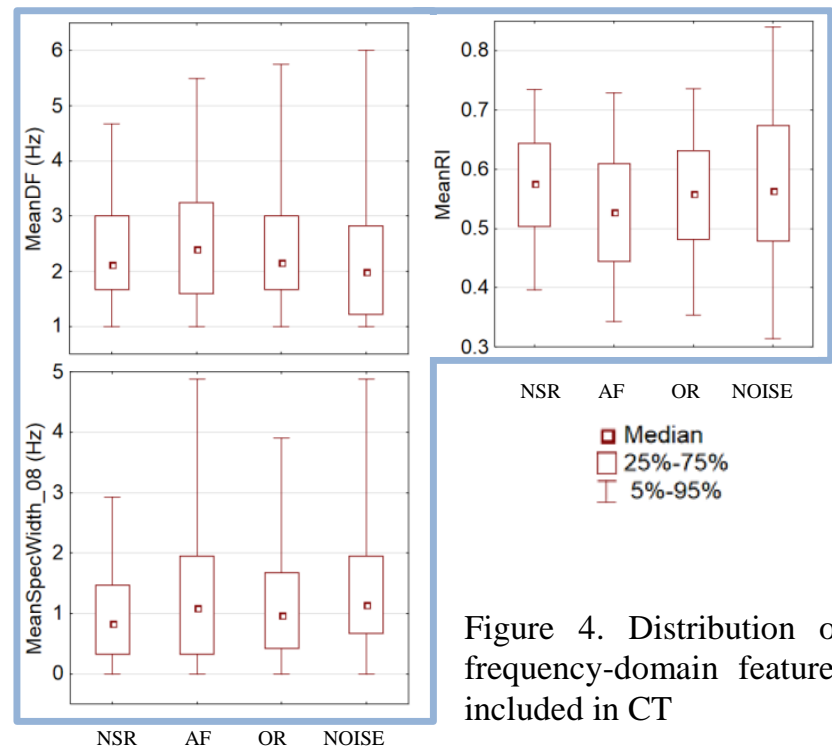

Figure 4. Distribution of frequency-domain features included in CT

\section{Results and Discussion}

The examples in Figure 2 illustrate the operation of the heartbeat detector, $\mathrm{P}$-wave detector and the calculation of two features specific for the designed module. Statistical distribution of the features involved in the CT design is presented in Figures 3,4. Evaluation of the Challenge entries is done by three rhythm specific ( $\left.F_{\text {RHYTHM }}\right)$ and one common (F1) score, calculated as:

$$
\begin{gathered}
\mathrm{F}_{\text {RHYTHM }}=\frac{2 . T P_{\text {RHYTHM }}}{T P_{\text {RHYTHM }}+F N_{\text {RHYTHM }}+F P_{\text {RHYTHM }}} \\
\mathrm{F} 1=\frac{F_{N S R}+F_{A F}+F_{O R}}{3}
\end{gathered}
$$

where TP, FN, FP stand for true positive, false negative, false positive detections. Our accuracy results, achieved over the training and a subset of the hidden test database are presented in Table 1 . The Challenge organizers assessed average running time about $10.3 \%$ of quota and score over the entire test set 0.64 (details for rhythm classification were not provided).

Table 1. Performance of the designed module for rhythm classification.

\begin{tabular}{|l|l|l|l|l|}
\hline \multirow{2}{*}{ Dataset } & \multicolumn{4}{l|}{ Scores } \\
\cline { 2 - 5 } & $\mathrm{F}_{\mathrm{NSR}}$ & $\mathrm{F}_{\mathrm{AF}}$ & $\mathrm{F}_{\mathrm{OR}}$ & $\mathrm{F} 1$ \\
\hline Training & 0.82 & 0.62 & 0.61 & 0.68 \\
\hline Test & 0.81 & 0.61 & 0.53 & 0.65 \\
\hline
\end{tabular}

There are two main reasons for the limited accuracy results: (i) the inconsistent annotations of the ECG signals, which obstruct the reliable training of the classifier; (ii) the wide variety of arrhythmia types in the OR class, which results in considerable overlapping between the calculated feature values for the couples (NSR, OR) and (AF, OR). This could be overcome via detailed analysis over the OR class and search for specific additional features for each of its subclasses.

\section{References}

[1] Naccarelli G, Varker H, Lin J, Schulman K. Increasing prevalence of atrial fibrillation and flutter in the United States. Am J Cardiol 2009;104(11):1534-9.

[2] Fuster V, Ryden L, Asinger R, Cannom D, Crijns H, Frye R, Halperin $\mathrm{J}$, et al. ACC/AHA/ESC guidelines for the management of patients with atrial fibrillation: executive summary. J Am Coll Cardiol 2001;38(4):1231-65.

[3] Du X, Rao N, Qian M, Liu D, Li J, Feng W, et al. A novel method for real-time atrial fibrillation detection in electrocardiograms using multiple parameters. Ann Noninvasive Electrocardiol 2014;19(3):217-25.

[4] Dotsinsky I. Atrial wave detection algorithm for discovery of some rhythm abnormalities. Physiol. Meas. 2007;28: 595-610.

[5] Ladavich S, Ghoraani B. Rate-independent detection of atrial fibrillation by statistical modeling of atrial activity. Biomed Signal Process Control 2015;18(4):274-81.

[6] Linker D. Accurate, Automated Detection of Atrial Fibrillation in Ambulatory Recordings. Cardiovasc Eng Technol 2016;7(2):182-9.

[7] Petrènas A, Marozas V, Sörnmo L. Low-complexity detection of atrial fibrillation in continuous long-term monitoring. Comput Biol Med 2015;65(10):184-91.

[8] Lake D, Moorman J. Accurate estimation of entropy in very short physiological time series: the problem of atrial fibrillation detection in implanted ventricular devices. Am J Physiol Heart Circ Physiol 2011;300(1):H319-H325.

[9] Babaeizadeh S, Gregg R, Helfenbein E, Lindauer J, Zhou S. Improvements in atrial fibrillation detection for real-time monitoring. J Electrocardiol 2009;42(6):522-6.

[10] Petrènas A, Sörnmo L, Lukoševicius A, Marozas V. Detection of occult paroxysmal atrial fibrillation. Med Biol Eng Comput 2015;53(4):287-97.

[11] Larburu N, Lopetegi T, Romero I. Comparative study of algorithms for atrial fibrillation detection. Computing in Cardiology 2011;38:265-8.

[12] Sasaki N, Okumura Y, Watanabe I, Madry A, Hamano Y, Nikaido $\mathrm{M}$, et al. Frequency analysis of atrial fibrillation from the specific ECG leads V7-V9: A lower DF in lead V9 is a marker of potential atrial remodelling. Journal of Cardiology 2015;66:388-94.

[13] Roonizi E, Sassi R. Dominant atrial fibrillatory frequency estimation using an extended Kalman smoother. Computing in Cardiology 2016;43: DOI:10.22489/CinC.2016.286-359.

[14] Dotsinsky I, Stoyanov T. Ventricular beat detection in single channel electrocardiograms. BioMed. Eng. Online 2004

[15] Zhang X, Zhu Y, Thakor N, Wang Z. Detecting ventricular tachycardia and fibrillation by complexity measure. IEEE Trans. Biomed. Eng. 1999;46:548-55.

[16] Kuo S, Dillman R. Computer detection of ventricular fibrillation. Comp. in Cardiol. 1978:347-9.

Address for correspondence.

Irena Jekova

Bulgarian Academy of Sciences,

Institute of Biophysics and Biomedical Engineering,

Acad. G. Bonchev str., bl.105, 1113, Sofia, Bulgaria

irena@biomed.bas.bg. 